\title{
Invertebrados asociados al coral constructor de arrecifes Pocillopora damicornis en Playa Blanca, Bahía Culebra, Costa Rica
}

\author{
Juan José Alvarado ${ }^{1,2} \&$ Rita Vargas-Castillo ${ }^{3}$ \\ 1. Centro de Investigación en Ciencias del Mar y Limnología (CIMAR), Universidad de Costa Rica, San Pedro, 11501- \\ 2060 San José, Costa Rica; juanalva76@yahoo.com \\ 2. Posgrado en Ciencias Marinas y Costeras, Universidad Autónoma de Baja California Sur, La Paz, México. \\ 3. Museo de Zoología, Escuela de Biología, Universidad de Costa Rica, San Pedro, 11501-2060 San José, Costa Rica; \\ rita.vargas@ucr.ac.cr
}

Recibido 07-IV-2011. C Corregido 23-I-2012. Aceptado 29-II-2012.

\begin{abstract}
Invertebrates associated with the reef-building coral Pocillopora damicornis at Playa Blanca, Bahía Culebra, Costa Rica. The coral reefs are one of the most diverse ecosystems in the planet, not only because their reef-building species but also because of the species that live above, on, inside and below them. Corals of the genus Pocillopora are recognized globally by harboring an important fauna of invertebrates among their branches, many of those considered obligate symbionts. The present investigation describes the fauna of invertebrates associated with the reef-building coral P. damicornis in Bahía Culebra, Costa Rica, their densities, frequencies, richness and diversities from 2003 to 2004. Five coral colonies were collected every 3-4 months at Playa Blanca within Bahía Culebra. A total of 448 individuals in 35 species were found: Harpiliopsis depressa, Trapezia ferruginea, Alpheus lottini, Fennera chacei, and Petrolisthes haigae, and the bivalve Lithophaga aristata, the predominant species. November was the month with the highest richness, diversity and taxonomic distinctiveness, while August had the lowest values. In general terms, the rainy season showed greater richness of species than the dry season. The species collected and their densities and abundances were similar to other sites along the Eastern Tropical Pacific. Bahía Culebra has been losing coral cover, which could have consequences on the diversity and abundances of associated corals organisms. These consequences include loss in the fertility of those organisms, a reduction in its function as cleaners and protection from coral predators, putting in risk their diversity, affecting the stocks of predatory fish that depends on them. Carrying out permanent monitorings of the associated cryptofauna of the coral Pocillopora is crucial to quantify losses or recoveries in the composition of associated invertebrates, and therefore in the functions that they perform in this ecosystem. Rev. Biol. Trop. 60 (Suppl. 2): 77-92. Epub 2012 April 01.
\end{abstract}

Key words: Decapoda, obligate symbionts, Costa Rica, coastal upwelling, conservation, Bahía Culebra.

Los arrecifes coralinos son considerados como uno de los ecosistemas más diversos que existen en el planeta (Reaka-Kudla 1997, Portes \& Tougas 2001, Glynn \& Enochs 2011), albergando entre 600000 y 9 millones de especies a nivel mundial (Knowlton 2001). Sin embargo, se cree que la verdadera diversidad de los arrecifes de coral es desconocida y que solo conocemos un $10 \%$ de ella (Sheppard et al. 2009). Entre los grupos más importantes destacan los peces, gasterópodos, bivalvos, crustáceos decápodos y equinodermos. Esta alta diversidad se debe no solo por la presencia de los corales pétreos que forman la estructura tridimensonal, sino también a la gran cantidad de organismos endo y ectosimbiontes presentes (Sheppard et al. 2009, Stella et al. 2011a), ya que los arrecifes coralinos son facilitadores de refugio y alimento a un gran número de peces e invertebrados (Idjadi \& Edmunds 2006).

En el Pacífico Tropical Oriental (PTO) los arrecifes coralinos están distribuidos desde el 
Golfo de California hasta Ecuador, incluyendo las islas oceánicas de Clipperton, Revillagigedo, Coco, Malpelo y Galápagos (Glynn \& Ault 2000, Cortés 2011). En términos generales son menos diversos que sus contrapartes del IndoPacífico o Caribe (Sheppard et al. 2009). Sin embargo, a pesar de ello siguen manteniendo cadenas tróficas tan complejas como los anteriores (Cortés 1997, Glynn 2004).

El estudio de la fauna asociada al coral Pocillopora spp. en el PTO se ha realizado principalmente entre los años 1974 y 1996 en Panamá (Abele 1974, 1976a, b, Castro 1974, Abele \& Patton 1976, Glynn 1976, 1983a, b, c, 1987, Castro 1978, 1996, Gotelli \& Abele 1983, Glynn et al. 1985, Gotelli et al. 1985) y Colombia (von Prahl et al. 1978, Castro 1982). Dichos trabajos se enfocaron inicialmente a la descripción de las especies de crustáceos simbiontes de los corales y posteriormente analizaron las relaciones ecológicas en las comunidades coralinas (Garth 1974, Castro 1976, 1982, 1996, von Prahl et al. 1978), para posteriormente entrar a procesos ecológicos que ellas juegan dentro del coral o arrecife. Abele (1976a, b) comparó la fauna de decápodos entre ambientes costeros y bajo condiciones oceanográficas diferentes, y Abele y Patton (1976) la relación entre el número de individuos por especie y el tamaño de la colonia. Glynn (1976) describió el proceso de defensa que hacen los simbiontes Trapezia y Alpheus ante la acción coralívora de la estrella corona de espinas, la cual prefiere atacar corales que no posean estos decápodos simbiontes. Por esta razón, se ha visto que la presencia de decápodos simbiontes favorece una mayor tasa de sobrevivencia en corales pocilopóridos al aumentar la producción de mucus y el crecimiento del esqueleto coralino, y liberarse de contaminantes, microorganismos, y estadios larvales (Glynn 1983). Sin embargo, los organismos simbiontes son afectados por la mortandad coralina (i.e. El Niño) al perder parte de su fuente de alimento (Glynn et al. 1985) de los cuales dependen completamente (Castro 1978). Posteriormente, Gotelli y Abele (1983) y Gotelli et al. (1985) estudiaron los patrones de estructura poblacional de varios de estos crustáceos asociados al coral Pocillopora damicornis. Más recientemente, Enochs y Hockensmith (2008) determinaron a través del tiempo que colonias vivas de $P$. damicornis siempre albergan una mayor biomasa de organismos crípticos que colonias muertas de este coral, resaltando la dependencia de estos organismo para habitar colonias vivas; y Enochs et al. (2011) determinan que la abundancia y biomasa de la criptofauna sobre este coral va a ser mayor en ambientes con baja porosidad y flujo de agua lenta, indicando que este tipo de ambientes van a brindar una mayor protección contra depredadores que ambientes muy porosos, mientras que un flujo lento va a ser sinónimo de ambientes con mayor deposición y retención de sedimentos y materia orgánica, favoreciendo una mayor biomasa.

En México este tipo de investigaciones comienza a darse a finales de los 1990, cuando Pereyra-Ortega (1998) y Hernández (1999) describieron la fauna de los decápodos asociados al coral en la Isla Espíritu Santo, Golfo de California. Posteriormente, Luna-Ramírez et al. (2002) estudiaron en Bahías de Huatulco la variación estacional de decápodos, encontrando que los meses lluviosos poseen los valores más bajos en abundancia y diversidad. Más recientemente, Hernández et al. (2008, 2010) analizaron el impacto de un huracán y el efecto del blanqueamiento coralino por bajas temperaturas en la composición de decápodos y otros invertebrados asociados al coral Pocillopora spp. en las bahías de Loreto y La Paz, Baja California Sur, y su posterior recuperación. Estos mismos autores presentaron un listado detallado de los crustáceos decápodos asociados a los arrecifes de Baja California Sur (Hernández et al. 2009).

Para Costa Rica no existen trabajos que analicen la fauna asociada al coral Pocillopora (Cortés \& Jiménez 2003, Cortés et al. 2010), por lo que los objetivos del presente trabajo fueron realizar un estudio de la fauna asociada a colonias del coral constructor de arrecifes, Pocillopora damicornis, en Playa Blanca, Bahía Culebra, con el fin de poder cuantificar cuales especies de invertebrados están presentes, en 
qué cantidades y la variación en la composición y abundancia de los organismos con respecto a la época del año. Esta bahía es una zona rica en arrecifes coralinos, con aproximadamente 16 especies de corales constructores de arrecife, y una cobertura de coral vivo de $44 \%$ (Jiménez 2001). El arrecife de Playa Blanca en los últimos 15 años ha venido sufriendo un deterioro en la cobertura de coral vivo, pasando de un 38\% (Jiménez 2001, Jiménez et al. 2001) a un $2.5 \%$ asociado a múltiples eventos de estrés que han causado una pérdida en la cobertura coralina (Alvarado et al. 2012).

\section{MATERIALES Y MÉTODOS}

El trabajo de campo se realizó en el arrecife de Playa Blanca $\left(10^{\circ} 36^{\prime} 37.63^{\prime \prime} \mathrm{N}\right.$, 8541'10.01'W), en la parte norte de Bahía Culebra. Esta bahía está sujeta a un afloramiento estacional de agua fría rica en nutrientes entre diciembre y abril (Brenes et al. 1990, Jiménez 2001), y consiste en una serie de islotes, playas, acantilados y estuarios con importantes recursos marinos económicos (Jiménez 2001). El presente estudio se realizó entre el
2003 y 2004, cuando la cobertura de coral todavía era alta y saludable. Para este periodo de trabajo las condiciones promedio de algunos parámetros ambientales fueron las siguientes: temperatura $\left(27.4 \pm 1.8^{\circ} \mathrm{C}\right.$, $\min : 22.6^{\circ} \mathrm{C}$, max: $\left.29.5^{\circ} \mathrm{C}\right)$, sedimentación $\left(4.5 \pm 4.3 \mathrm{mg} / \mathrm{cm}^{2} /\right.$ día, min: $1.0 \mathrm{mg} / \mathrm{cm}^{2} /$ día, $\max : 16.3 \mathrm{mg} / \mathrm{cm}^{2} /$ día) y concentración de fosfatos $(0.16 \pm 0.06 \mu \mathrm{M}$, min: $0.06 \mu \mathrm{M}$, max: $0.28 \mu \mathrm{M}$ ) (Fig. 1; datos sin publicar, Laboratorio de Arrecifes Coralinos, CIMAR-UCR). Las recolectas realizadas en este estudio se llevaron a cabo los días 30 abril 2003, 31 agosto 2003, 21 noviembre 2003 y 25 febrero 2004; entre las 10:00 y 14:00 horas.

Durante cada visita se recolectaron cinco colonias del coral Pocillopora damicornis que tuvieran un diámetro aproximado de $20 \mathrm{~cm}$, con morfologías similares, y que no presentaran signos de deterioro o blanqueamiento, de manera azarosa a $3 \mathrm{~m}$ de profundidad mediante equipo de buceo autónomo. Las colonias fueron puestas en bolsas plásticas, sellándolas al nivel de la base de la colonia, y posteriormente llevadas a la costa donde fueron quebradas con el fin de remover todos los invertebrados presentes en ellas. Los organismos fueron

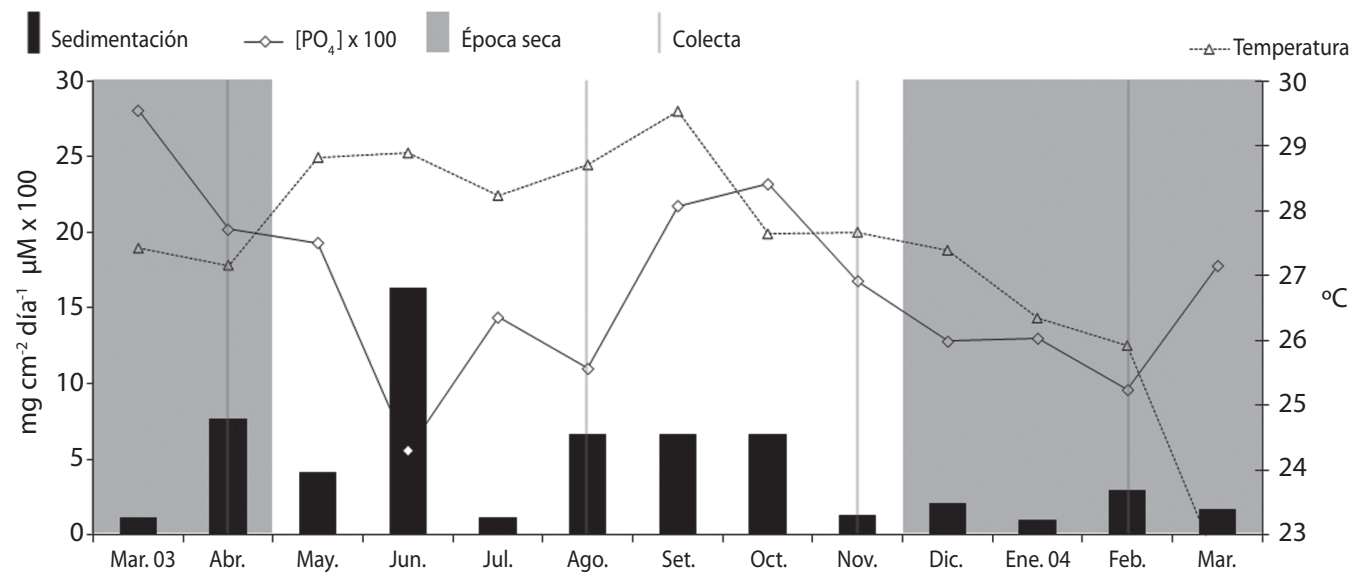

Fig. 1. Comportamiento de la temperatura promedio $\left({ }^{\circ} \mathrm{C}\right.$; línea punteada con triángulos blancos), tasa de sedimentación promedio ( $\mathrm{g} / \mathrm{cm}^{2} /$ día; barras negras), y concentración de fosfato promedio multiplicado por $100\left(\mu \mathrm{M} \mathrm{PO}_{4}{ }^{3-}\right.$; línea negra continua con rombos blancos) en Playa Blanca, Bahía Culebra, entre marzo 2003 y marzo 2004. Sombreado gris: época seca o de afloramiento.

Fig. 1. Behavior of the average temperature $\left({ }^{\circ} \mathrm{C}\right.$; pointed line with white triangles), mean sedimentation rates $\left(\mathrm{g} / \mathrm{cm}^{2} / \mathrm{day}\right.$; black bars), phosphate concentration multiplied by $100\left(\mu \mathrm{M} \mathrm{PO}_{4}{ }^{3-}\right.$; continuous black line with white diamonds) in Playa Blanca, Bahía Culebra, between March 2003 and March 2004. Gray shadow: dry season or upwelling season. 
separados en frascos y preservados en alcohol al 70\%. Posteriormente, los organismos fueron trasladados al Museo de Zoología, Escuela de Biología (Universidad de Costa Rica), donde se realizó la identificación. La identificación siempre que fuera posible, se llevó hasta el nivel de especie siguiendo los criterios de Keen (1971) y Skoglund $(1992,2002)$ para los gasterópodos y bivalvos, Holthuis (1951, 1952), Haig (1960), Kim y Abele (1988) y Castro (1996) para los decápodos, Salgado-Barragan y Hendrickx (2010) para los estomatópodos, y Clark (1940) para los ofiuroideos.

Para cada especie se presenta la cantidad de individuos por colonia por mes de muestreo, así como el promedio anual de individuos por colonia de cada especie. Así mismo, para cada especie se calculó su densidad (ind $/ \mathrm{m}^{2}$ ) al dividir el número de individuos por colonia, por el área de la colonia, asumiendo como diámetro de las colonias $20 \mathrm{~cm}$, con el fin de poder realizar comparaciones de densidad con otras zonas del Pacífico Tropical Oriental. Así mismo, con el fin de determinar la predominancia de las especies a través del año se empleó el índice de valor biológico de Sanders (IVB; Sanders 1960, Loya-Salinas \& Escofet 1990). Para este índice, se designaron como especies predominantes, aquellas que a lo largo del año fueran dominantes en cada mes de muestreo, y que presentaron las mayores sumas acumuladas del IVB mensuales con respecto a las otras especies, seleccionando las primeras diez con mayor puntaje del índice.

Con el fin de determinar si existían diferencias significativas entre la cantidad de individuos por mes muestreado se realizaron una serie de pruebas pareadas de $\mathrm{T}$ de Student mediante el programa estadístico SYSTAT 8.0. Así mismo, con el fin de determinar si existían diferencias entre el número de especies y de individuos entre época seca (abril 2003 y febrero 2004) y época lluviosa (agosto y noviembre 2003), se realizaron dos pruebas de Chi-cuadrado utilizando el programa estadístico PAST. Como complemento a estas pruebas se realizó un análisis de similitud porcentual (SIMPER), con el fin de determinar cuáles especies contribuyen mayormente a las posibles diferencias entre épocas del año. Para esta prueba, se utilizaron los datos de abundancia, los cuales fueron estandarizados y posteriormente transformados con la función de $\log _{10}(x+1)$. Este análisis se realizó utilizando el software PRIMER 6.0 (Clarke \& Gorley 2006). Así mismo, con el fin de determinar similitudes en la composición de especies, se construyó una matriz de similitud basada en las distancias entre curvas de dominancias de especies utilizando la rutina DOMDIS de PRIMER 6.0 (Clarke 1990, Clarke \& Gorley 2006). Con esta matriz se construyó un dendrograma de agrupamiento promedio y un escalamiento multidimensional no métrico (nMDS). Posteriormente, se realizó un análisis de similitud (ANOSIM) para determinar el grado de significancia de las diferencias entre los grupos (Clarke \& Gorley 2006).

Para cada mes de muestreo se calcularon los índices de riqueza de especies de Margalef (d), de diversidad de Shannon $\left(\mathrm{H}^{\prime}, \log _{\mathrm{e}}\right.$ ), la equidad de Pielou (J) y la diferenciación taxonómica $(\Delta+$; Clarke \& Warwick 2001). Para éste último índice se utilizaron seis niveles taxonómicos: especie, género, familia, orden, clase, y filo; con sus respectivos pesos $\omega=$ 16.66 (especies en el mismo género), 33.33 (misma familia pero géneros diferentes), 50 (mismo orden pero familia diferente), 66.66 (misma clase pero orden diferente), 83.33 (mismo Filo pero clase diferente) y 100 (filos diferentes). Estos análisis se llevaron a cabo utilizando el software PRIMER 6.0 (Clarke \& Gorley 2006).

\section{RESULTADOS}

De las 20 colonias muestreadas de $P$. damicornis se recolectó un total de 448 individuos pertenecientes a 35 especies (Cuadro 1), 28 géneros, 24 familias, 11 órdenes, cinco clases y tres filos. Noviembre 2003 fue el mes con mayor número de especies e individuos (23 y 131 respectivamente), mientras que febrero 2004 fue el que menos especies e individuos presentó (15 y 91 respectivamente; Cuadro 2). Sin embargo, no se observaron diferencias 


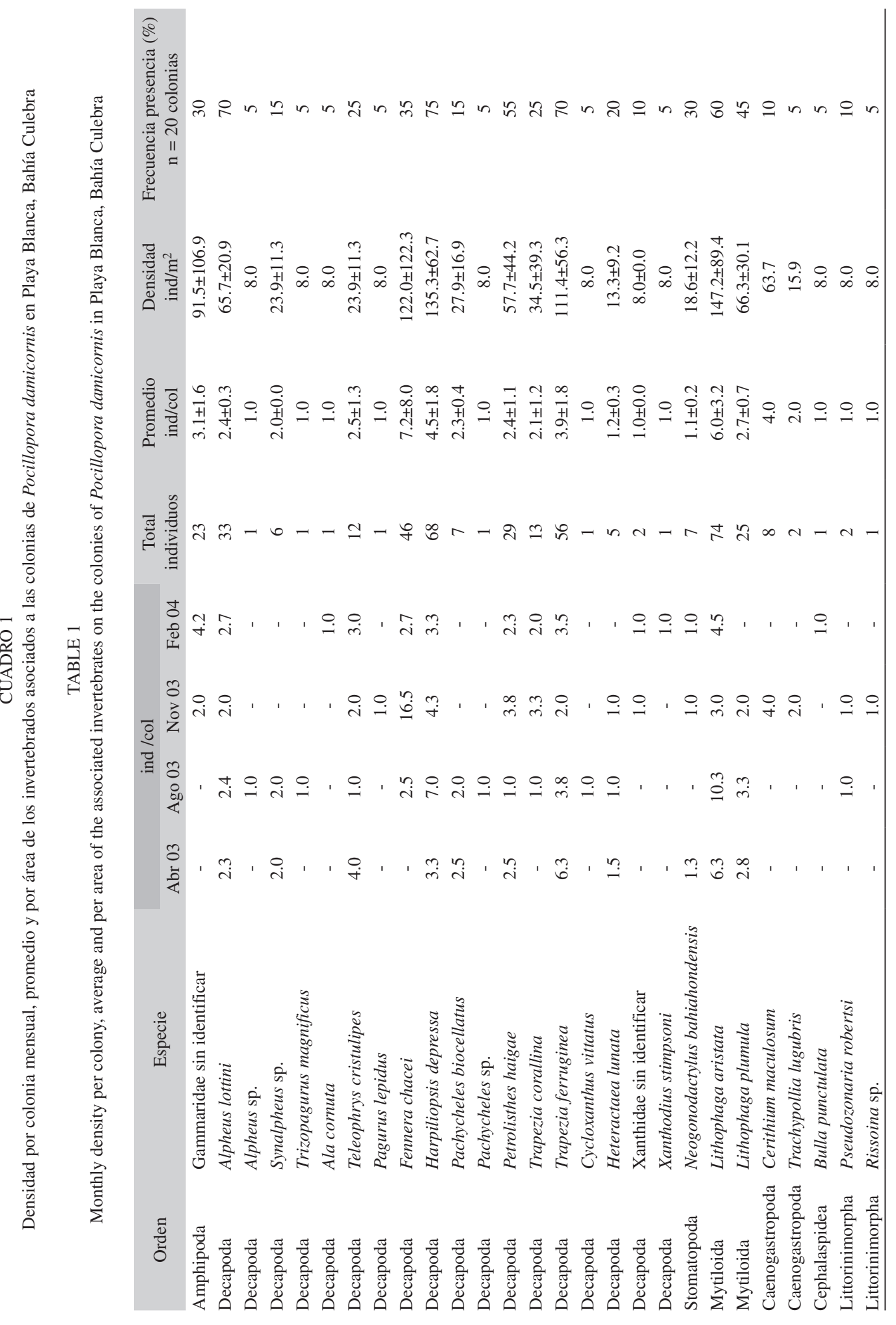




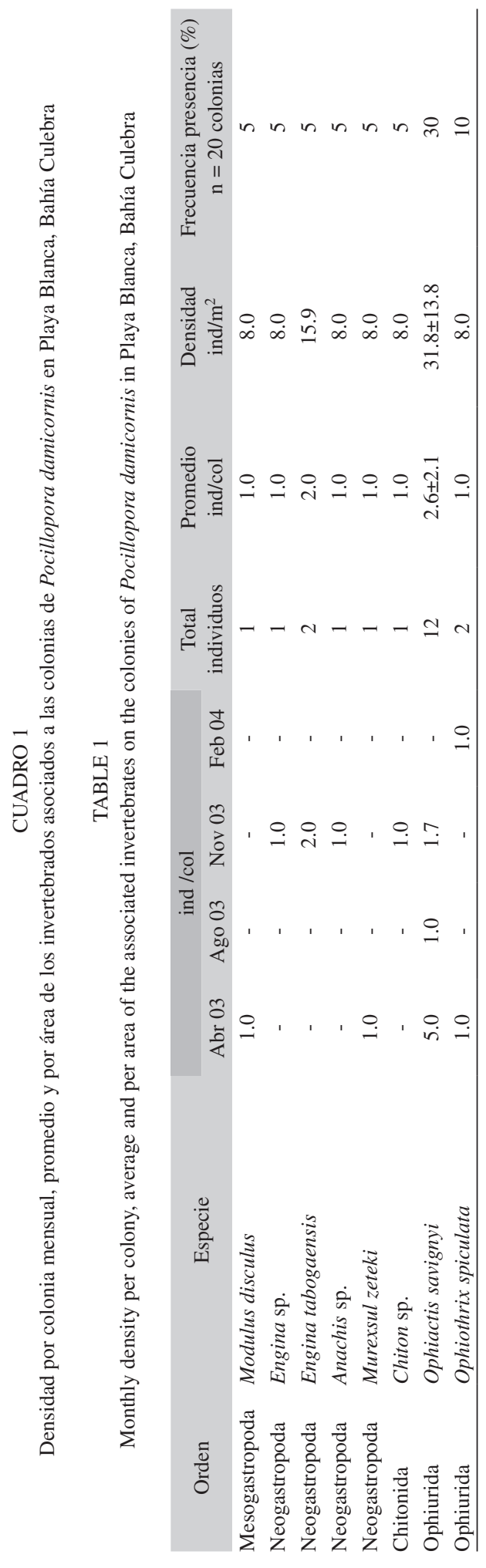

significativas en la composición de especies entre los meses (Cuadro 3). Así mismo, la época lluviosa (agosto y noviembre 2003) fue la que presentó el mayor número de especies y de individuos (29 y 254 respectivamente). Mientras que la época seca (abril 2003 y febrero 2004) menos organismos presentó (22 y 194 respectivamente). En este caso, se observaron diferencias significativas entre el total de individuos por época del año $\left(\chi^{2}=8.04, \mathrm{gl}=1\right.$, $\mathrm{P}<0.05)$, pero no entre el número de especies por época $\left(\chi^{2}=0.96, \mathrm{gl}=1, \mathrm{P}=0.33\right)$.

Las especies con mayor número de individuos fueron Lithophaga aristata con 74 individuos, Harpiliopsis depressa con 68 y Trapezia ferruginea con 56. Estas tres especies más Alpheus lottini y Petrolisthes haigae fueron las únicas que estuvieron presentes en todas las fechas de muestreo. El decápodo Fennera chacei y el bivalvo L. aristata fueron las especies con mayor densidad de individuos por colonia a lo largo del año de estudio con $7.2 \pm 8.0$ y $6.0 \pm 3.2 \mathrm{ind} /$ colonia, seguidos por los decápodos $H$. depressa (4.5 \pm 1.8 ind/colonia) y

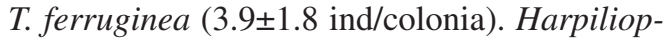
sis depressa fue la especie que apareció con mayor frecuencia en las colonias recolectadas, apareciendo en un $75 \%$ de ellas (Cuadro 1), seguida por A. lottini y T. ferruginea con $70 \%$ de ocurrencia, y L. aristata y $P$. haigae con un $60 \%$ y $55 \%$ respectivamente. A nivel de densidad por $\mathrm{m}^{2}$, estas especies siguen teniendo los números más altos, sin embargo el orden cambia un poco siendo $L$. aristata la que posee la mayor densidad con $147.2 \pm 89.4 \mathrm{ind} / \mathrm{m}^{2}$, seguida por $H$. depressa $\left(135.3 \pm 62.7 \mathrm{ind} / \mathrm{m}^{2}\right)$, F. chacei $\left(122.0 \pm 122.3 \mathrm{ind} / \mathrm{m}^{2}\right)$ y $T$. ferruginea (111.4 $\pm 56.3 \mathrm{ind} / \mathrm{m}^{2}$; Cuadro 1$)$.

Así mismo, el índice de valor biológico de Sanders (IVB) indicó que las diez especies que predominaron durante todo el estudio fueron: H. depressa y L. aristata con un $13.9 \%$ ambas, seguidas por T. ferruginea (11.8\%), A. lottini (9.7\%), F. chacei (8.9\%), P. haigae (8.4\%), Lithophaga plumula (6.8\%), Ophiactis savignyi (4.6\%), un Gammaridae sin identificar (4.2\%) y Trapezia corallina (3.8\%). El resto de especies presentaron valores del índice por 
CUADRO 2

Total de especies (S), total de individuos (N), riqueza de especies de Margalef (d), diversidad de Shannon (H'), equidad de Pielou (J'), y diferenciación taxonómica $(\Delta+)$ por mes de colecta de invertebrados en colonias de Pocillopora damicornis en Playa Blanca, Bahía Culebra

TABLE 2

Total species (S), total individuals (N), Margalef species richness (d), Shannon diversity (H'),

Pielou's evenness $\left(\mathrm{J}^{\prime}\right)$, and Taxonomic distinctiveness $(\Delta+)$ per sampling month of the associated invertebrates on the colonies of Pocillopora damicornis in Playa Blanca, Bahía Culebra

\begin{tabular}{lcccccc}
\multicolumn{1}{c}{ Fecha } & S & N & d & H' & J' & $\Delta+$ \\
Abr. 03 & 15 & 103 & 3.021 & 2.311 & 0.8533 & 69.93 \\
Ago. 03 & 18 & 123 & 3.533 & 2.159 & 0.7471 & 58.45 \\
Nov. 03 & 23 & 131 & 4.513 & 2.550 & 0.8133 & 71.15 \\
Feb. 04 & 15 & 91 & 3.104 & 2.252 & 0.8315 & 60.82 \\
\hline
\end{tabular}

\section{CUADRO 3}

Pruebas pareadas de T de Student de la composición de invertebrados asociados por mes de colecta en colonias de Pocillopora damicornis en Playa Blanca, Bahía Culebra

TABLE 3

Paired T-student test of the monthly composition of the associated invertebrates on the colonies of Pocillopora damicornis in Playa Blanca, Bahía Culebra

\begin{tabular}{lcc}
\multicolumn{1}{c}{ Prueba } & $\mathrm{T}$ & $\mathrm{P}$ \\
Mar-03 vs Ago-03 & -0.91 & 0.37 \\
Mar-03 vs Nov-03 & -0.62 & 0.54 \\
Mar-03 vs Feb-03 & 0.374 & 0.71 \\
Ago-03 vs Nov-03 & -0.17 & 0.86 \\
Ago-03 vs Feb-03 & 0.86 & 0.39 \\
Nov-03 vs Feb-03 & -1.09 & 0.28 \\
Seca vs Lluviosa & -1.44 & 0.16 \\
\hline
\end{tabular}

debajo del 3\%. El análisis de similitud porcentual (Cuadro 4), indicó que las especies $T$. ferruginea, $L$. aristata, y $H$. depressa fueron las que mayor porcentaje aportaron en la diferenciación en época seca, mientras que para la época lluviosa fueron de nuevo $H$. depressa, $L$. aristata y A. lottini (Cuadro 4).

Los meses de época seca (abr 03 y feb 04) fueron los más similares (80\%), mientras que los de época lluviosa (ago 03 y nov 03) no formaron alguna agrupación (Fig. 2 A, B), siendo significativas estas separaciones (ANOSIM: R $=0.5$, nivel de significancia $33.3 \%$ ). Noviembre $\mathrm{y}$ agosto fueron los meses que compartieron más especies (12), mientras que abril-agosto y noviembre-febrero compartieron 11 especies cada una, abril-noviembre 10 especies, y abril-febrero y agosto-febrero compartieron 8 especies. Noviembre presentó ocho especies que solo ocurrieron en ese mes, mientras que en agosto cuatro, febrero tres y abril dos. De esta manera 13 especies solo aparecieron en la época lluviosa (Alpheus sp., Anachis sp., Cerithium maculosum, Chiton sp., Cycloxanthus vittatus, Pseudozonaria robertsi, Engina sp., Engina tabogaensis, Trachypollia lugubris, Pachycheles sp., Pagurus lepidus, Rissoina sp. y Trizopagurus magnificus), mientras que en la época seca solo seis especies aparecieron (Ala cornuta, Bulla punctulata, Modulus disculus, Muricopsis zeteki, Xanthodius stimpsoni, Ophiothrix spiculata) (Cuadro 1).

Con respecto a la riqueza de especies de Margalef (d) noviembre 2003 fue el que presentó los valores más altos, mientras que abril 2003 los más bajos (Cuadro 2). De la misma manera, de acuerdo al índice de diversidad de Shannon (H') noviembre 2003 fue el más diverso, y agosto 2003 como el menos diverso. Abril 2003 presentó los mayores valores de la equidad de Pielou ( $\mathrm{J}$; 0.8533), mientras que agosto 2003 de nuevo alcanzó los valores más bajos (0.7471). Finalmente, con respecto al índice diferenciación taxonómica $(\Delta+)$ se repitió el mismo patrón, siendo noviembre 2003 el mes con mayor valor (71.15) y agosto 2003 con los valores más bajos (58.42) (Cuadro 2). 


\section{CUADRO 4}

Resultados del análisis de similitud porcentual (SIMPER) indicando las especies que contribuyen a la diferenciación entre épocas del año

TABLE 4

Results of the similarity percentage analysis (SIMPER) indicating species contribution to the difference between seasons of the years

\begin{tabular}{lcccc}
\multicolumn{1}{c}{ Época seca } & Abundancia promedio & Similitud promedio & Contribución (\%) & Acumulativo (\%) \\
Trapezia ferruginea & 2.88 & 10.98 & 19.62 & 19.62 \\
Lithophaga aristata & 2.81 & 9.38 & 16.75 & 36.37 \\
Harpiliopsis depressa & 2.55 & 9.31 & 16.63 & 53.01 \\
Alpheus lottini & 2.17 & 8.07 & 14.41 & 67.41 \\
Petrolisthes haigae & 1.96 & 6.94 & 12.4 & 79.81 \\
Teleophrys cristulipes & 1.52 & 5.73 & 10.23 & 90.04 \\
\multicolumn{1}{c}{ Época lluviosa } & & & 17.47 & 17.47 \\
Harpiliopsis depressa & 2.9 & 9.61 & 13.67 & 31.14 \\
Lithophaga aristata & 2.66 & 7.52 & 11.39 & 42.52 \\
Alpheus lottini & 2.05 & 6.26 & 10.75 & 53.27 \\
Fennera chacei & 2.44 & 5.91 & 9.27 & 62.54 \\
Lithophaga plumula & 1.81 & 5.1 & 9.27 & 71.81 \\
Trapezia ferruginea & 2.1 & 5.1 & 6.4 & 78.21 \\
Ophiactis savignyi & 1.27 & 3.52 & 6.4 & 84.60 \\
Petrolisthes haigae & 1.74 & 3.52 & 3.94 & 88.54 \\
Teleophrys cristulipes & 1 & 2.17 & 3.94 & 92.49 \\
Trapezia corallina & 1.38 & 2.17 & & \\
\hline
\end{tabular}

Al analizar la contribución porcentual de individuos por familia de organismo asociado a $P$. damicornis por mes (Fig. 3) se notaron varios patrones. La contribución de la familia de bivalvos Mytilidae es diferente porcentualmente entre abril-agosto (35-33\%) y noviembre-febrero (10\%). La familia de decápodos Palaemonidae presentó bajos porcentajes (10-23\%) en época seca (abril y febrero), en comparación con los meses lluviosos (agosto-noviembre) donde sus porcentajes fueron mayores (27-38\%). Las familias de decápodos Trapeziidae, Majidae, Porcellanidae y Xanthidae, así como la familia de estomatópodo Gonodactylidae, fueron bastante constantes porcentualmente en el número de individuos a través del tiempo. Además de estos aparentes patrones, la familia Gammaridae fue casi nula durante todo el muestreo con la excepción de febrero donde mostró una alta contribución $(23 \%)$. Hubo varias familias (Buccinidae,
Bullidae, Cerithiidae, Columbellidae, Cypraeidae, Diogenidae, Mithracidae, Modullidae, Muricidae, Ophiothricidae, Paguridae, Polyplacophora, Rissoidae, y Thaidinae) cuya contribución porcentual fue muy baja (1-2\%), y que además solo se presentaron en uno o dos meses de muestreo. En este sentido noviembre fue el mes donde hubo un mayor porcentaje $(14 \%)$ de estas familias poco frecuentes.

\section{DISCUSIÓN}

En el presente estudio se encontraron 35 especies de invertebrados asociados a $P$. damicornis, de las cuales 20 fueron crustáceos, 13 moluscos y dos equinodermos. La mayor parte de estudios realizados en el PTO tratan sobre la fauna de decápodos asociados, y pocos son los que presentan información de otros grupos. Por lo tanto, parte de esta discusión se centrará en el grupo de decápodos, no sin antes recalcar la 


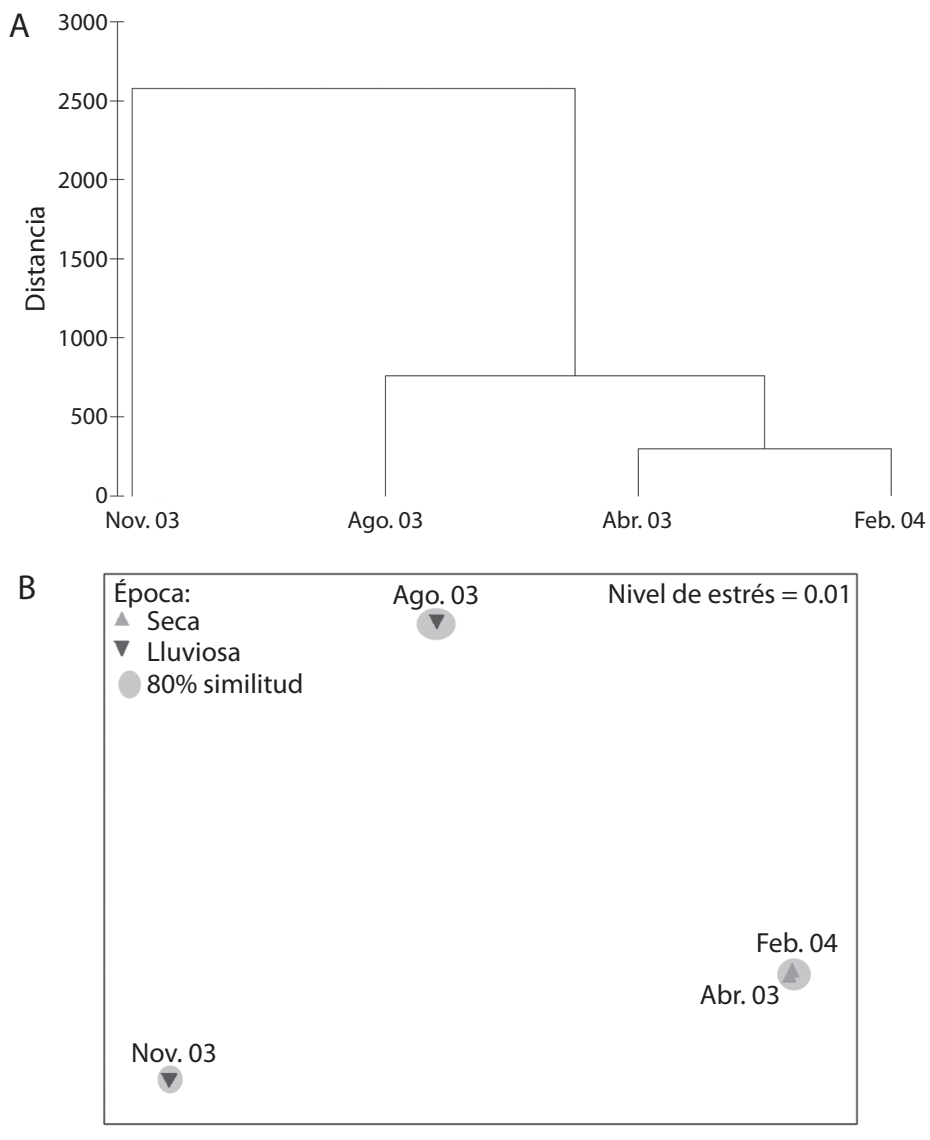

Fig. 2. A) Dendrograma de unión simple y B) escalamiento multidimensional no métrico (nMDS) establecidos de una matriz de similitud basada en las distancias entre curvas de dominancias de especies utilizando la rutina DOMDIS. ANOSIM: R = 0.5 , nivel de significancia $=33.3 \%$.

Fig. 2. A) Simple union cluster tree and B) Non-metric Multi-Dimensional Scaling (nMDS) established from a similitude matrix based on the Distance between species dominance curves using the routine DOMDIS. ANOSIM: $\mathrm{R}=0.5$, significance level $=33.3 \%$.

necesidad de realizar mayores investigaciones que abarquen toda la fauna asociada a este tipo de corales.

Entre estos pocos trabajos que incluyen otros organismos tenemos el de Hernández et al. (2008) para dos localidades del Golfo de California, México, donde encontraron 50 especies asociadas a Pocillopora, entre equinodermos, crustáceos, moluscos y poliquetos, contabilizando todos los organismos presentes en el arrecife, y no solo los que habitan dentro de las colonias. De estas 50 especies,
19 (Bahía de la Paz) y 15 (Bahía de Loreto) fueron decápodos asociados a las colonias de coral (Hernández et al. 2010). Así mismo, en Panamá también dominó el grupo de los decápodos (96\% Golfo de Panamá, y 89\% Golfo de Chiriquí) entre toda la macrofauna asociada a las colonias de coral (Abele \& Patton 1976).

Abele y Patton (1976) y Gotelli y Abele (1983) encontraron que la abundancia de individuos y la cantidad de especies de decápodos está correlacionado positivamente con el tamaño de las colonias de coral estudiadas. 


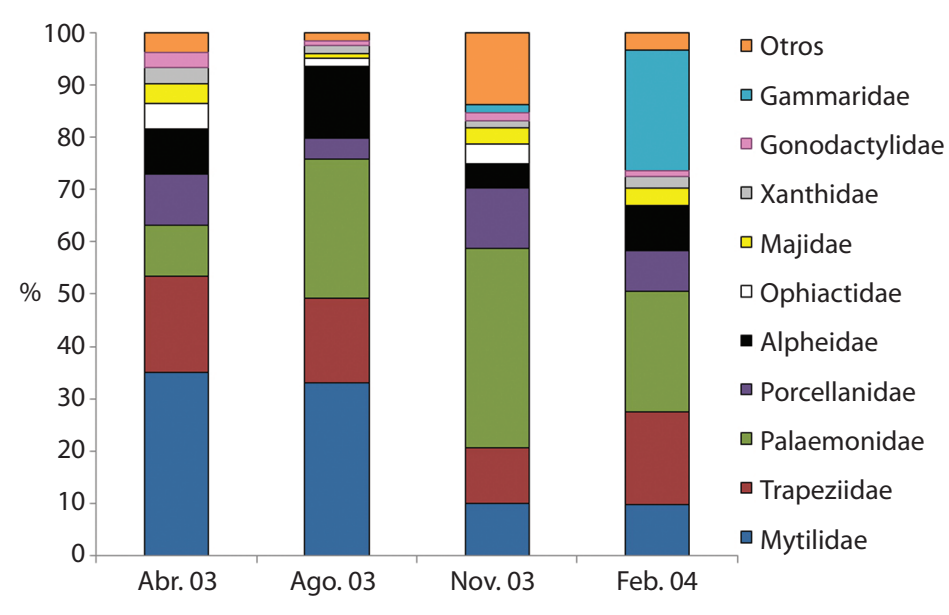

Fig. 3. Contribución porcentual por familia al total de especies recolectadas por mes asociadas al coral Pocillopora damicornis en Bahía Culebra. La categoría de "otros" integra familias que solo aparecieron una o dos veces con muy pocos individuos.

Fig. 3. Percentage contribution per family to the total of species sampled per month associated to the coral Pocillopora damicornis in Bahía Culebra. The "otros (others)" category integrated families that only appeared one or two times with few individuals.

El número se incrementa de tres especies en colonias de $960 \mathrm{~cm}^{3}$ a 22 especies en colonias de $22500 \mathrm{~cm}^{3}$ (Abele \& Patton 1976). En nuestra investigación, para el caso de decápodos, encontramos 18 especies con un tamaño aproximado de colonia de $25000 \mathrm{~cm}^{3}$. Entre estas 18 especies se encuentran todos los simbiontes obligatorios de coral (Luna-Ramírez et al. 2002, Hernández et al. 2010). Abele (1974, 1976a) registró 55 especies de decápodos asociados a Pocillopora en las Islas Perlas en el Golfo de Panamá y 37 en la Isla de Uva en el Golfo de Chiriquí, encontrando las mismas especies obligatorias del coral, especies frecuentes y otras poco comunes. Posteriormente, para el arrecife de Uva, Gotelli y Abele (1983) mecionaron la presencia de 52 especies de decápodos en 119 colonias de coral. Abele (1976a) también analizó 119 colonias de coral, mientras que en el presente estudio se analizaron 20 en total, por lo cual podríamos pensar que de aumentar el esfuerzo de muestreo este número podría incrementarse notablemente. Sin embargo, la metodología debería modificarse para que al aumentar el esfuerzo no aumente la modificación del paisaje, pasando más bien a censos visuales con transectos como los realizados por Hernández et al. (2008, 2209, 2010) en México, complementándolo con extracción de colonias, ya que extraer 120 colonias de $20 \mathrm{~cm}$ de diámetro implicaría una fuerte alteración al fondo marino.

Por otro lado la cantidad de especies asociadas a Pocillopora en el PTO es menor a la determina en otras especies de corales ramosos en el Indo-Pacifico. Stella et al. (2010) encuentran 178 especies asociadas a cuatro especies de coral: Acropora nasuta, A. millepora, $P$. damicornis y Seriatopora hystrix. Esta última especie fue la que presentó la mayor cantidad de especies (105) seguida por $P$. damicornis (102 especies). Así mismo, el grupo de los crustáceos decápodos fue el que dominó las muestras, representando entre el 51-66\% de la fauna examinada. Stella et al. (2010) argumentan que este mayor numero de especie en $S$. hystrix y $P$. damicornis es producto un patrón de crecimiento de las ramas más complejo, el cual brinda mayor protección contra depredación. 
De las especies encontradas en el presente estudio podemos destacar seis especies (cinco decápodos y un bivalvo) como las predominantes de acuerdo a su densidad (tanto por colonia como por $\mathrm{m}^{2}$ ), su frecuencia de presencia y su índice de valor biológico, las cuales fueron en orden de predominancia (de mayor a menor): Harpiliopsis depressa, Lithophaga aristata, Trapezia ferruginea, Alpheus lottini, Fennera chacei, y Petrolisthes haigae. Todas consideradas como simbiontes obligatorios de P. damicornis (Luna-Ramírez et al. 2002, Hernández et al. 2010), con la excepción de L. aristata. Estas mismas especies han sido las más comunes en Gorgona (Colombia; von Prahl et al. 1978, Castro 1982), Uva (Golfo de Chiriquí, Panamá; Gotelli \& Abele 1983, Enochs \& Hockensmith 2008), las Perlas (Golfo de Panamá, Panamá; Abele \& Patton 1976), Huatulco (México; Luna-Ramírez et al. 2002), y Loreto y Espíritu Santo (Golfo de California, México; Hernández et al. 2008, 2009, 2010). Así mismo, las densidades (Cuadro 1) presentes en este trabajo son muy similares a las observadas en todas estas localidades del PTO.

En la Isla Gorgona, Colombia, Castro (1982) mencionó que la cantidad de individuos de Trapezia estaba correlacionada significativamente con el área de las colonias. En este sentido, Castro (1982) enfatizó que T. ferruginea estuvo presente en 18 de las 20 colonias analizadas con densidades entre 1 y 6 ind/ colonia, siendo la segunda en importancia para este género. Trapezia corallina fue la especie más abundante apareciendo en 19 de las 20 colonias con densidades mayores, entre 2 y 16 ind/colonia (Castro 1982). En Bahía Culebra, esta especie de Trapezia tuvo muy baja frecuencia de aparición en las colonias (25\%) con densidades inferiores ( $2.1 \pm 1.2 \mathrm{ind} /$ colonia) a las informadas para Gorgona. Así mismo, para esta última localidad el camarón A. lottini estuvo presente en el $100 \%$ de las colonias analizadas. Glynn (1976) halló para el arrecife de Uva una densidad de $T$. ferruginea entre 2 y $20 \mathrm{ind} /$ colonia, siendo estos más numerosos en el flanco del lado del mar ("seaward flank") en el arrecife. Por su parte, encuentra densidades menores (2-4 ind/colonia) para $A$. lottini. Esta especie estuvo presente en $80 \%$ de las colonias analizadas (Gotelli et al. 1985). Para esta misma localidad, utilizando las mismas muestras que Glynn (1976), Gotelli et al. (1985) reportan densidades para $F$. chacei entre 1.7 y 5.3 ind/colonia, apareciendo en el $72 \%$ de las colonias, mientras que $H$. depressa estuvo presente en $92 \%$ de las colonias. En Huatulco, México, Luna-Ramírez et al. (2002) indicaron que $T$. ferruginea y $P$. haigae estuvieron presentes en el $100 \%$ de los meses muestreados (desde marzo 1994 hasta abril 1995) siendo a su vez las más abundantes.

Durante el presente estudio noviembre presentó los valores más altos de los índices de riqueza de especies (d), diversidad de Shannon $\left(\mathrm{H}^{\prime}\right)$ y diferenciación taxonómica $\left(\Delta^{+}\right)$(Cuadro 2). Así mismo, fue el mes menos similar en comparación con el resto de los meses (Fig. 2), aportando nuevas especies o exclusivas (8). Durante este mes, se observó la menor tasa de sedimentación $\left(1.3 \mathrm{mg} / \mathrm{cm}^{2} /\right.$ día $)$, una alta disponibilidad de nutrientes $\left(0.16 \mu \mathrm{M} \mathrm{PO}_{4}^{-3}\right)$, y una temperatura cálida $\left(27.7^{\circ} \mathrm{C}\right)$ en el arrecife, condiciones que pudieron favorecer esta alta diversidad y riqueza, y la separación del resto de meses. Por otro lado, en el mes de agosto se presentaron condiciones similares en temperatura $\left(28.7^{\circ} \mathrm{C}\right)$ y de nutrientes $\left(0.11 \mu \mathrm{M} \mathrm{PO}_{4}^{-3}\right)$, fue el mes que presentó los menores valores de diversidad ( $\left.\mathrm{H}^{\prime}\right)$, equidad (J') y diferenciación taxonómica $\left(\Delta^{+}\right)$, distanciándose de noviembre y de los meses de época seca. Sin embargo, fue uno de los meses con mayor tasa de sedimentación $\left(6.6 \mathrm{mg} / \mathrm{cm}^{2} /\right.$ día $)$, después de abril (7.6 $\mathrm{mg} / \mathrm{cm}^{2} /$ día), indicando que este último factor pudo haber tenido cierto efecto en la composición de especies. Febrero y abril, a pesar de poseer leves diferencias en temperatura $\left(25.9^{\circ} \mathrm{C}\right.$ y $27.2^{\circ} \mathrm{C}$, respectivamente), nutrientes $(0.09$ $\mu \mathrm{M} \mathrm{PO}_{4}{ }^{-3}$ y $0.20 \mu \mathrm{M} \mathrm{PO}_{4}{ }^{-3}$, respectivamente), y sedimentación $\left(2.9 \mathrm{mg} / \mathrm{cm}^{2} /\right.$ día y $7.6 \mathrm{mg} / \mathrm{cm}^{2} /$ día, respectivamente), fueron los más similares en composición (Fig. 2). Esto indica que deben de haber factores ambientales que no tomamos en cuenta en el presente estudio que expliquen esta agrupación de meses que corresponden a la 
época seca, que definitivamente está asociado con algún evento relacionado al afloramiento costero. Posiblemente, eventos de reclutamiento posteriores a la época de afloramiento costero se manifiestan en el arrecife, por lo que en meses como noviembre podamos encontrar más especies. Al respecto, Lessios (1981) argumentó que el erizo de mar Diadema mexicanum, en el Golfo de Panamá, posee picos de desove en época lluviosa con el fin de asegurase que sus larvas no sean dispersadas fuera del arrecife y que se asienten previo al afloramiento cuando hay mayor disponibilidad de alimento.

De acuerdo a Gotelli et al. (1985) la época del año es un factor importante que influencia la estructura poblacional de los decápodos asociados a coral. Ellos encuentran que en la época de lluvias la salinidad disminuye, y la escorrentía incrementa la turbidez del agua y reduce la penetración de la luz afectando la composición. Para el Golfo de Chiriquí (Panamá), una zona sin afloramiento costero, Gotelli y Abele (1983) encuentran que las densidades y riquezas de especies no difirieron entre época seca y lluviosa. Abele (1976a) compara esta misma zona, con el Golfo de Panamá que sufre afloramiento costero al igual que Bahía Culebra, y encuentra que en la zona con condiciones ambientales más fluctuantes, la riqueza de especies es mayor. Por su parte, Luna-Ramírezet al. (2002) en Bahía de Huatulco, zona de afloramiento, estudiaron la variación espacio temporal a lo largo de 19 meses, encontrando que la época seca posee los mayores valores de riqueza, abundancia y diversidad, mientras que la época de lluvias los más bajos. Estos patrones difieren con lo encontrando en la presente investigación, lo cual debe tomarse con cautela en nuestro caso, ya que el tamaño de muestra (cinco colonias por mes) y las fechas estuvieron muy dispersas. Probablemente el factor más determinante en nuestro caso fueron los meses de recolecta ya que varios estuvieron en momentos de transición entre una época y otra, por lo que debería aumentarse el número de meses por época de repetirse una investigación como esta.
Por otro lado, la fauna de crustáceos decápodos y otros invertebrados asociados al coral Pocillopora disminuyen su riqueza, abundancia y diversidad de especies luego de eventos de huracanes (Hernández et al. 2008) o blanqueamiento coralino (Glynn 1983b, Hernández et al. 2010). Posterior al evento de un huracán en el 2006 en las bahías de Loreto y la Paz, la fauna pasó de 50 especies a 39 (Hernández et al. 2008). Durante un evento de blanqueamiento coralino por aguas frías en el 2008, la fauna de decápodos se mantuvo relativamente estable en La Paz, mientras que en Loreto disminuyó su riqueza, abundancia y diversidad, permitiendo inclusive un cambio en la fauna asociada obligatoria al coral (Hernández et al. 2010). Este tipo de estrés causa la disminución en la producción de mucus por parte del coral del cual dependen los decápodos simbiontes (Gotellli \& Abele 1983). Así mismo, si los simbiontes de Pocillopora son removidos, la producción de mucus disminuye, las paredes de los pólipos se desintegran y ocurre exfoliación masiva del tejido (Glynn 1983a), y al ocurrir blanqueamiento, las colonias de coral disminuyen su número de simbiontes de manera considerable (Glynn 1983b), convirtiéndose en una relación altamente simbiótica y delicada. Si a esto le añadimos la disminución en cobertura coralina que se viene sufriendo a nivel global, estos hallazgos nos van sugerir que la pérdida en cobertura y estructura coralina van a conllevar una pérdida sustancial en la diversidad de invertebrados (Idjadi \& Edmunds 2006). La mortalidad coralina reduce la biomasa de la fauna asociada, disminuyendo por lo tanto los grupos tróficos que dependen de ella directa e indirectamente, llevando a un colapso en las funciones ecológicas del arrecife (Enochs \& Hockensmith 2008). Niveles extremos de mortalidad coralina, bioerosión y destrucción del hábitat van a llevar a la discapacidad y eventual perdida de las funciones del ecosistema (Enochs 2012). Así mismo, el blanqueamiento coralino produce una disminución sustancial en el tamaño de los huevos de los cangrejos simbiontes, provocando una reducción en su fecundidad, tenido como consecuencia una 
reducción en su función como limpiadores y protectores de depredadores del coral (Stella $e t$ al. 2011b). Finalmente, Pocillopora es uno de los géneros de coral a nivel mundial que albergan una mayor cantidad de especies asociadas de invertebrados, pero que a su vez una de las más susceptibles a los efectos del blanqueamiento, poniendo en riesgo esa alta diversidad que mantiene si las condiciones se vuelven desfavorables para el coral (Stella et al. 2011a). Enochs (2012) indica que la desaparición de la criptofauna asociada a Pocillopora por muerte coralina va a tener serias implicaciones en los stocks de peces depredadores que dependen de estos organismos durante alguna etapa de su vida. Esta criptofauna puede persistir luego de eventos de mortalidad coralina, manteniendo su papel en la dinámica arrecifal. Sin embargo bajo largos periodos de bioerosión y destrucción del basamento coralino estas poblaciones van a colapsar al ir perdiendo la protección que les daba la estructura arrecifal (Enochs 2012).

Este hecho es de suma importancia para la zona de Bahía Culebra, la cual ha venido sufriendo de varios disturbios, como propagaciones de macroalgas (Fernández \& Cortés 2005, Fernández 2007, Fernández-García et al. 2012), mareas rojas (Morales-Ramírez et al. 2001, Vargas-Montero et al. 2008), alta bioerosión por erizos de mar (Alvarado et al. 2012), que están produciendo una alta mortalidad coralina (Jiménez et al. 2001, Cortés et al. 2010, Alvarado et al. 2012, Jiménez et al. en prep.), y por consiguiente una posible pérdida en la biodiversidad de los organismos asociados a los arrecifes coralinos. Por lo tanto, se hace necesario y urgente realizar monitoreos de este tipo en Bahía Culebra con el fin de determinar si ha ocurrido un cambio notable en la composición de invertebrados asociados a estos arrecifes, o se ha dado algún tipo de recuperación. Así mismo, es necesario profundizar este tipo de investigación para determinar si existen patrones de reclutamiento o cierta posible estacionalidad (Fig. 3) en la composición de la fauna asociada no simbionte obligatoria.

En conclusión, se puede decir que la fauna de invertebrados asociados a $P$. damicornis en Bahía Culebra, entre el 2003 y 2004, fue muy similar a la observada en otras zonas del PTO, compuesta por los mismos simbiontes obligatorios y en proporciones muy similares. Sin embargo, debido al acelerado deterioro ambiental que está sufriendo esta zona del país, es posible que la diversidad de organismos se esté perdiendo, y con ello eliminar las funciones ecológicas que ayudan a la resiliencia del arrecife. Por lo tanto, es urgente la toma de medidas de manejo que mejoren las condiciones ambientales y permitan una pronta recuperación de estos ecosistemas. La fauna de invertebrados, y sobre todo la de decápodos, pueden funcionar como indicadores de esta recuperación, tomando en cuenta este trabajo como línea base.

\section{AGRADECIMIENTOS}

El presente trabajo no se hubiera podido realizar sin la cooperación y ayuda en el campo y laboratorio de C. Fernández, D. Morera, J. Nivia, J. Cortés, E. Salas, B. Bezy, E. Ruíz, y A. Sánchez. Así mismo, queremos agradecer el apoyo que nos brindó Ecodesarrollo Papagayo, el Instituto Costarricense de Turismo, el Consejo Nacional para Investigaciones Científicas y Tecnológicas de Costa Rica y el Consejo Nacional de Ciencia y Tecnología de México. Finalmente extendemos un especial agradecimiento a J. Cortés y dos revisores anónimos que con sus comentarios enriquecieron este trabajo.

\section{RESUMEN}

Los arrecifes coralinos son uno de los ecosistemas más diversos en el planeta, tanto por los organismos constructores como por aquellos que viven encima, sobre, dentro y debajo de ellos. Los corales del género Pocillopora son reconocidos mundialmente por albergar una importante fauna de invertebrados entre sus ramas, los cuales son considerados como simbiontes obligatorios en una gran cantidad de casos. La presente investigación describe la fauna de invertebrados asociados al coral Pocillopora damicornis en Bahía Culebra, Costa Rica, describiendo sus densidades, frecuencias, riquezas y diversidades a través del tiempo. Para esto se colectaron 5 colonias cada 3-4 meses en Playa Blanca, Bahía Culebra. En total se encontraron 448 individuos en 35 especies, siendo Harpiliopsis depressa, 
Lithophaga aristata, Trapezia ferruginea, Alpheus lottini, Fennera chacei, y Petrolisthes haigae las especies predominantes. Noviembre fue el mes en el que se encontraron los mayores valores en los índices de riqueza, diversidad y diferenciación taxonómica, mientras que agosto fue el que presentó los valores más bajos de todos. En términos generales, la época lluviosa mostró mayor riqueza de especies que la época seca. Así mismo, las especies colectados y los valores obtenidos son muy similares a otras zonas del Pacífico Oriental Tropical. Culebra ha venido sufriendo una pérdida en la cobertura coralina, que podría tener consecuencias en la diversidad y abundancia de organismos asociados a corales. Estas consecuencias incluyen pérdida en la fecundidad de estos organismos, una reducción en su función como limpiadores y protectores de depredadores del coral, poniendo en riesgo su diversidad, lo que puede afectar los stocks de peces depredadores que depende de ellos. Realizar monitoreos permanentes de la criptofauna asociada al coral Pocillopora va a ser determinante para cuantificar pérdidas o recuperaciones en la composición de invertebrados asociados, y por ende en las funciones que estos desempeñan en este ecosistema.

Palabras claves: Decápoda, simbiontes obligatorios, Costa Rica, afloramiento costero, conservación, Bahía Culebra.

\section{REFERENCIAS}

Abele, L.G. 1974. Species diversity of decapod crustaceans in marine habitats. Ecology 55: 156-161.

Abele L.G. 1976a. Comparative species richness in fluctuating and constant environments: coral-associated decapod crustaceans. Science 192: 461-463.

Abele, L.G. 1976b. Comparative species composition and relative abundance of decapod crustaceans in marine habitats of Panamá. Mar. Biol. 38: 263-278.

Abele, L.G. \& W.K. Patton. 1976. The size of coral heads and the community biology of associated decapod crustaceans. J. Biogeogr. 3: 35-47.

Alvarado J.J., J. Cortés \& H. Reyes-Bonilla. 2012. Reconstruction of Diadema mexicanum bioerosion impact on three Costa Rican Pacific coral reefs. Rev. Biol. Trop. 60 (Suppl. 2): 121-132.

Brenes, C.L., S. León, A. Gutiérrez \& G. Arroyo. 1990. Condiciones hidrográficas en la región de los "Papagayos". Rev. Geofísica 33: 5-19.

Castro, P. 1976, Brachyuran crabs symbionts with scleractinian corals: a review of their biology. Micronesia 12: 99-110.
Castro, P. 1978. Movements between coral colonies in Trapezia ferruginea (Crustacea: Brachyura), on obligate symbiont of scleractinian corals. Mar. Biol. 46: 237-245.

Castro, P. 1982. Notes on symbiotic decapod crustaceans from Gorgona Island, Colombia, with a revision of the eastern pacific species Trapezia (Brachyura, Xanthidae), symbionts of scleractinian corals. An. Inst. Inv. Mar. Punta de Betín 12: 9-17.

Castro, P. 1996. Eastern Pacific species of Trapezia (Crustacea, Brachyura: Trapeziidae) sibling species symbiotic with reef corals. Bull. Mar. Sci. 58: 531-554.

Clark, H.L. 1940. Eastern Pacific Expeditions of the New York Zoological Society. XXI. Notes on Echinoderms from the west coast of Central America. Zoologica 25: 331-352.

Clarke, K.R. 1990. Comparisons of dominance curves. J. Exp. Mar. Biol. Ecol. 138: 143-157.

Clarke, K.R. \& R.N. Gorley. 2006. PRIMER v6: User manual. PRIMER-E Ltd, Plymouth, U.K.

Clarke, K.R. \& R.M. Warwick. 2001. A further biodiversity index aplicable to species list: variation in taxonomic distinctness. Mar. Ecol. Prog. Ser. 216: 265-278.

Cortés, J. 1997. Biology and geology of eastern Pacific coral reefs. Coral Reefs 16 (Suppl.): S39-S46.

Cortés, J. \& C.E. Jiménez. 2003. Corals and coral reefs of the Pacific of Costa Rica: history, research and status, p. 361-385. In: J. Cortés (Ed.). Latin American Coral Reefs. Elsevier Science B.V., Amsterdam.

Cortés, J., C.E. Jiménez, A.C. Fonseca \& J.J. Alvarado. 2010. Status and conservation of coral reefs in Costa Rica. Rev. Biol. Trop. 58 (Suppl. 1): 33-50.

Enochs, I.C. 2012. Motile cryptofauna associated with live and dead coral substrates: implications for coral mortality and framework erosion. Mar. Biol.: DOI 10.1007/s00227-011-1848-7

Enochs, I.C. \& G. Hockensmith. 2008. Effects of coral mortality on the community composition of cryptic metazoans associated with Pocillopora damicornis. Proc. $11^{\text {th }}$ Int. Coral Reef Symp., Ft. Lauderdale: 1375-1379.

Enochs, I.C., L.T. Toth, V.W. Brandtneris, J.C. Afflerbach \& D.P. Manzello. 2011. Environmental determinants of motile cryptofauna on a Eastern Pacific coral reef. Mar. Ecol. Prog. Ser. 438: 105-118. 
Fernández, C. 2007. Propagación del alga Caulerpa sertularioides en Bahía Culebra, Golfo de Papagayo, Pacífico norte de Costa Rica. Tesis de Maestría, Universidad de Costa Rica, San José, Costa Rica.

Fernández, C. \& J. Cortés. 2005. Caulerpa sertularioides, a green alga spreading aggressively over coral reef communities in Culebra Bay, North Pacific of Costa Rica. Coral Reefs 24: 10

Fernández-García, C., Cortés, J., Alvarado, J.J. \& NiviaRuiz, J. 2012. Physical factors contributing to the benthic dominance of the alga Caulerpa sertularioides (Caulerpaceae, Chlorophyta) in the upwelling Bahía Culebra, north Pacific of Costa Rica. Rev. Biol. Trop. 60 (Suppl. 2): 93-107.

Garth, J.S. 1974. On the occurrence in the Eastern Tropical Pacific of Indo-West Pacific decapods crustaceans commensal with reef-building corals. Proc. $2^{\text {th }}$ Int. Coral Reef Symp., Brisbane 1: 397-404.

Glynn, P.W. 1976. Some physical and biological determinants of coral community structure in the Eastern Pacific. Ecol. Monogr. 46: 431-456.

Glynn, P.W. 1983a. Increased survivorship in corals harboring crustacean symbionts. Mar. Biol. Let. 4: 105-111.

Glynn, P.W. 1983b. Extensive bleaching and death of reef corals on the Pacific coast of Panama. Environ. Conserv. 10: 149-154.

Glynn, P.W. 1983c. Crustacean symbionts and the defense of corals:coevolution on the reef?, p. 111-178. In: M.H. Nitecki (ed.). Coevolution. Universityof Chicago Press, Chicago.

Glynn, P.W. 1987. Some ecological consequences of coralcrustacean guard mutualisms in the Indian and Pacific Oceans. Symbiosis 4:303-324.

Glynn, P.W. 2004. High complexity food webs in lowdiversity Eastern Pacific reef-coral communities. Ecosystems 7:358-367.

Glynn, P.W. \& J.S. Ault. 2000. A biogeographic analysis and review of the far eastern Pacific coral reef region. Coral Reefs 19:1-23.

Glynn, P.W. \& I.C. Enochs. 2011. Invertebrates and their roles in coral reef ecosystems, p. 273-325. In: Z Dubinsky \& N. Stambler (eds.). Coral Reefs: An Ecosystem in Transition. Springer, Berlin.

Glynn, P.W., M. Pérez \& S.L. Gilchrist. 1985. Lipid decline in stressed corals and their crustaceans symbionts. Biol. Bull. 168: 276-284.
Gotelli, N.J. \& L.G. Abele. 1983. Community patterns of coral-associated decapods. Mar. Ecol. Prog. Ser. 13: 131-139.

Gotelli, N.J., S.L. Gilchrist \& L.G. Abele. 1985. Population biology of Trapezia spp. and other coral-associated decapods. Mar. Ecol. Prog. Ser. 21: 89-98.

Haig, J. 1960. The Porcellanidae (Crustacea: Anomura) of the eastern Pacific. Allan Hancock Pac. Exped. 24: $1-440$

Hernández, L. 1999. Camarones stenopodideos y carideos (Crustacea: Decapoda: Stenopodidae, Caridea) asociados a colonias de coral, en los Islotes, B.C.S., México. Tesis Licenciatura, Universidad Autónoma de Baja California Sur, La Paz, México. 51 p.

Hernández, L., E.F. Balart \& H. Reyes-Bonilla. 2008. Effect of hurricane John (2006) on the invertebrates associated with corals in Bahia de la Paz, Gulf of California. Proc. $11^{\text {th }}$ Int. Coral Reef Symp., Ft. Lauderdale: 301-304.

Hernández, L., E.F. Balart \& H. Reyes-Bonilla. 2009. Checklist of reef decapods crustaceans (Crustacea: Decapoda) in the southern Gulf of California, México. Zootaxa 2119: 39-50.

Hernández, L., H. Reyes-Bonilla \& E.F. Balart. 2010. Efecto del blanqueamiento del coral por baja temperatura en los crustáceos decápodos asociados a arrecifes del suroeste del Golfo de California. Rev. Mex. Biodiv. 81: S113-S119.

Holthuis, L.B. 1951. A general revision of the Palaemonidae (Crustacea: Decapoda: Natantia) of the Americas. I. The subfamilies Euryrhynchinae and Pontoniinae. Occ. Pap. Allan Hancock Found. 11:1-332.

Holthuis, L.B. 1952. A general revision of the Palaemonidae (Crustacea: Decapoda: Natantia) of the Americas, Part. II. The subfamily Palaemoninae. Occ. Pap. Allan Hancock Found.12: 1-396.

Idjadi, J.A. \& P.J. Edmunds. 2006. Scleractinian corals as facilitators for other invertebrates on a Caribbean reef. Mar. Ecol. Prog. Ser. 319: 117-127.

Jiménez, C. 2001. Arrecifes y ambientes coralinos de Bahía Culebra, Pacífico de Costa Rica: aspectos biológicos, económico-recreativos y de manejo. Rev. Biol. Trop. 49 (Supl. 2): 215-231.

Jiménez, C., J. Cortés, A. León \& E. Ruiz. 2001. Coral bleaching and mortality associated with the1997-98 El Niño in an upwelling environment in the eastern Pacific (Gulf of Papagayo, Costa Rica). Bull. Mar. Sci. 69: 151-169. 
Keen, A.M. 1971. Sea Shells of Tropical West America: Marine Molluscs from Baja California to Peru. Stanford University, Stanford.

Kim, W. \& L.G. Abele. 1988. The snapping shrimp genus Alpheus from the Eastern Pacific (Decapoda: Caridea: Alpheidae). Smithson. Contr. Zool. 454: 1-119.

Knowlton, N. 2001. The future of coral reefs. Proc. Nat. Acad. Sci. USA. 98: 541-5425.

Loya-Salinas, D.H. \& A. Escofet. 1990. Aportaciones al cálculo del índice de valor biológico (Sanders, 1960). Cienc. Mar. 16: 97-115.

Lessios, H.A. 1981. Reproductive periodicity of the echinoid Diadema and Echinometra on the two coasts of Panama. J. Exp. Mar. Biol. Ecol. 50: 47-61.

Luna-Ramírez, S., G. de la Cruz \& N. Barrientos-Luján. 2002. Variación espacio temporal de Porcellanidae, Majoidea y Xanthoidea asociados a los corales del género Pocillopora en Bahías de Huatulco, México, p. 233-254. In: M.E. Hendricks (ed.). Contribuciones al Estudio de los Crustáceos del Pacífico Este. Vol. 1. UNAM, ICMYL, Mazatlán, México.

Morales-Ramírez, A., R. Víquez, K. Rodríguez \& M. Vargas. 2001. Marea roja producida por Lingulodinium polyedrum (Peridiniales, Dinophyceae) en Bahía Culebra, Golfo de Papagayo, Costa Rica. Rev. Biol. Trop. 49 (Supl. 2): 19-23.

Pereyra-Ortega, R.T. 1998. Cangrejos anomuros y braquiuros (Crustacea: Decapoda) simbiontes del coral Pocillopora elegans de los Islotes, Baja California Sur, México. Tesis Licenciatura, Universidad Autónoma de Baja California Sur, La Paz, México. 94 p.

Porter, J.W. \& J.J. Tougas. 2001. Reef ecosystems: threats to their biodiversity. Encyclop. Biodiver. 5: 73-95.

Reaka-Kudla, M.L. 1997. The global biodiversity of coral reefs: a comparison with rain forest, p. 83-108. In: M.L. Reaka-Kudla, D.E. Wilson, \& E.O. Wilson (eds.). Biodiversity II. Joseph Henry Press, Washington, D.C.

Salgado-Barragán, P. \& M. Hendrickx. 2010. Clave ilustrada para la identificación de los estomatópodos (Crustacea:Hoplocarida) del Pacífico oriental. Rev. Mex. Biod. 81: 1-49.

Skoglund, C. 1992. Additions to the Panamic province gastropod (Mollusca) literature 1971 to 1992 . Festivus 24: 1-169.

Skoglund, C. 2002. Panamic province molluscan literature: additions and changes from 1971 through 2001. III Gastropoda. Festivus 32: 201-286.

Sanders, H.L. 1960. Benthic studies in Buzzards Bay. III. The structure of the soft-bottom community. Limnol. Oceanogr. 5: 138-153.

Sheppard, C.R.C, S.K. Davy \& G.M. Pilling. 2009. The Biology of Coral Reefs. Oxford University Press, New York. 339 p.

Stella, J.S., G.P. Jones \& M.S. Pratchett. 2010. Variation in the structure of epifaunal invertebrate assemblages among coral hosts. Coral Reefs 29: 957-973.

Stella, J.S., M.S. Pratchett, P.A. Hutchings \& G.P. Jones. 2011a. Coral-associated invertebrates: diversity, ecological importance and vulnerability to disturbance. Oceanogr. Mar. Biol. An. Rev. 49: 43-104.

Stella, J.S., P.L. Munday \& G.P. Jones. 2011b. Effects of coral bleaching on the obligate coral-dwelling crab Trapezia cymodoce. Coral Reefs 30: 719-727.

Vargas-Montero, M., E. Freer-Bustamante, J.C. Guzmán \& J.C. Vargas. 2008. Florecimientos de dinoflagelados nocivos en la costa Pacífica de Costa Rica. Hidrobiológica 18 (Supl. 1): 15-23.

Von Prahl, H., F. Guhl \& M. Grögl. 1978. Crustáceos decápodos comensales del coral Pocillopora damicornis L. en la Isla de Gorgona, Colombia. A. Inst. Inv. Mar. Punta Betín 10: 81-93. 